\title{
CALCULANDO MOBILIDADE ATIVA PARA 0 BRASIL: O CASO DE BELO HORIZONTE, MG
}

\section{CALCULATING WALKABILITY FOR BRAZIL: A CASE STUDY FOR BELO HORIZONTE, MG}

\section{SOUZA, RENATO CÉSAR FERREIRA DE}

Ph.D Architecture -UK, Post Graduate Certificate in Higher Education PCHE -UK

Professor Associado da Escola de Arquitetura da Universidade Federal de Minas Gerais e-mail: rcesar@arq.ufmg.br

\section{RESUMO}

Discute-se a metodologia para a determinação de indicadores de Mobilidade Ativa (MA) em cidades brasileiras, confrontando-se padrões internacionais e adaptações requeridas para o Brasil. Toma-se a cidade de Belo Horizonte como estudo de caso da pesquisa ainda em curso. Argumenta-se que o estudo dos dados secundários do espaço urbano, quando primeiramente realizado, permite intuir com mais profundidade questões para entrevistas e questionários que capturem, quantitativamente, os dados primários para compreender a maneira como a população percebe a utilidade, a segurança, 0 conforto e 0 interesse de suas caminhadas pela cidade. Um estudo de caso é apresentado, indicando-se as etapas para o cálculo do índice de Mobilidade Ativa para uma cidade brasileira, e são explicadas as suas dificuldades e abstrações. O mapa final de índices de MA é analisado e criticado, e o encaminhamento das próximas etapas da pesquisa é sugerido. Finalmente, algumas estratégias são enfatizadas como ferramental útil para cidades brasileiras que não ainda possuem dados secundários consolidados em um sistema de informações geográficas.

PALAVRAS-CHAVE: mobilidade Ativa; Mobilidade urbana; Índices e métricas urbanas.

\section{RESUMEN}

Se discute la metodología para la determinación de indicadores de Movilidad Activa (MA) en ciudades brasileñas, confrontándose estándares internacionales y adaptaciones requeridas para Brasil. Se toma la ciudad de Belo Horizonte como estudio de caso de la investigación aún en curso. Se argumenta que el estudio de los datos secundarios del espacio urbano, cuando se realiza primero, permite intuir con más profundidad cuestiones para entrevistas y cuestionarios que capturen, cuantitativamente, los datos primarios para comprender la manera como la población percibe la utilidad, la seguridad, confort y el interés de sus caminatas por la ciudad. Un estudio de caso se presenta, indicando las etapas para el cálculo del índice de Movilidad Activa para una ciudad brasileña, y se explican sus dificultades y abstracciones. El mapa final de índices de MA es analizado y criticado y el encaminamiento de las próximas etapas de la investigación es sugerido. Finalmente, algunas estrategias son enfatizadas como herramientas útiles para ciudades brasileñas que no aún poseen datos secundarios consolidados en un sistema de informaciones geográficas.

PALABRAS-CLAVE: movilidad activa; Mobilidad urbana; Índices y métricas urbanas.

\section{ABSTRACT}

This article discusses the methodology for measurement of Walkability Indicators in Brazilian cities, confronting it with international standards and introducing the adaptations required for Brazil. It takes the city of Belo Horizonte as a partial case- study not accomplished so far. It is argued that the study of secondary data in urban space, when previously done, allows more accurately insights to create questions for interviews and questionnaires that quantitatively capture the primary data to understand how the population perceives utility, safety, Comfort and the interest of its walks through the city. A case study is presented, indicating the steps for calculating the Walkability Index for a Brazilian city, and explaining its difficulties and abstractions. The final map of Walkability indexes is analyzed and criticized, and future search is suggested. Finally, some strategies are emphasized as useful tool for Brazilian cities that do not yet have consolidated secondary data in a geographic information system.

KEYWORDS: walkability; urban mobility; indexes and urban metrics.

\section{INTRODUÇÃO}

Na última década, “Walkability” passou a ser tema agregado às problemáticas contemporâneas, sobretudo na relação do cidadão com o uso dos espaços públicos das metrópoles em todo mundo. Refletindo-se no Brasil, ela vem congregando diferentes atores e concentrando diversos tipos de esforços com a finalidade de se tornar parte da agenda pública com sua mensagem principal: quando o cidadão anda, faz bem a si e à cidade. Com essa mensagem simples, o termo "Walkability" foi abrasileirado com nomes estranhos, como "caminhabilidade", "andabilidade", e outros, que não expressam o que se trata e a sua complexidade. "Walkability" será traduzida neste artigo pela expressão "Mobilidade Ativa" (MA), retirada das disciplinas de Cineantropometria e Desempenho Humano, da Educação Física (LIMA; RECH; REIS, 2013; MALAVASI et alt., 2007).

Mobilidade Ativa é um dos elementos estratégicos que fazem parte da mobilidade urbana em geral, estudando o movimento do pedestre através dos meios físicos que o suportam, estudando a condição física e a manutenção de sua saúde, caracterizando os aspectos qualitativos sobre o quê o motiva a caminhar e 
as vantagens coletivas que a MA podem trazer para a transformação da vida da cidade em uma vida urbana mais sustentável.

Discute-se aqui uma adaptação metodológica para o início da pesquisa de MA nas cidades brasileiras, apresentando o experimento do teste metodológico como um estudo de caso. Conclui-se sobre as idiossincrasias metodológicas, suas vantagens para os métodos de observação social sistemática e as possibilidades de adaptações para cidades onde faltem dados completos para o cálculo.

\section{QUESTÕES METODOLÓGICAS}

Brian Saeles e James Sallis (2002) criaram o "Neighborhood Environmental Walkability Scale" (News), talvez o primeiro conjunto estruturado de perguntas e observações para avaliar a MA sob o ponto de vista da percepção da população de subúrbios americanos. Tentavam capturar como os moradores classificavam o espaço urbano em que viviam em termos de como percebiam as atividades, densidade residencial, acessibilidade e proximidade ao comércio e outros usos, conectividade das vias, infraestrutura para caminhadas e para o ciclismo, tráfego, segurança contra crimes, bem como a satisfação individual. Esse método foi utilizado e adaptado em diversos países e desde então, múltiplas versões do questionário original estão disponíveis.

Atualmente o professor Sallis sinaliza, em seu site, que além das entrevistas, o método deve ter seus resultados discutidos à luz dos Sistemas de Informações geográficas (SIG), na versão metodológica que ele chamou News-CFA (SALLIS, 2003). Com forte orientação para as ciências sociais, mas aproximando-se da saúde pública, o método NEWS e sua evolução demonstraram a necessidade de uma abordagem transdisciplinar no estudo da MA. No entanto, foi gradativa a aproximação dos campos de conhecimento e o engendramento de quadros teóricos e metodológicos apropriados para tratar as complexidades da urbanização contemporânea.

Shay Et. al. comenta que, até 2003, a literatura a respeito da Mobilidade Ativa era confusa, imprecisa e incapaz de fornecer elementos para estudos sistemáticos, localizados e válidos. As pesquisas limitavam-se a comparar conceitos de acessibilidade entre si, e enumerar hipotéticas características que seriam capazes de contribuir para a criação de caminhos de pedestres que favorecessem a saúde, a economia local, a sustentabilidade (SHAY et al., 2003) e até o nível acadêmico dos indivíduos (LUFKIN, 2016). Não havia, àquela época, nem um preciso conceito do termo e nem muito menos um bem definido conjunto de correlações entre o comportamento universal de pedestres e os aspectos espaciais do ambiente construído.

Com o crescimento do mundo urbanizado e suas consequentes dificuldades, os desafios para a Mobilidade Ativa conseguiram impor o seu reconhecimento para agendas em todo mundo. A Organização Mundial de Saúde promoveu, em 10 de maio de 2002, o dia Mundial da Saúde cujo tema foi "Mobilidade para Saúde" (WHO, 2010). Gradativamente, o espectro das interferências do espaço urbano sobre a saúde das populações foi aumentando nas abordagens de MA. O meio ambiente construído e as formas sociais nele ancoradas passaram a ser estudadas como agentes recursivamente interferentes que sofrem interferências da modulação de tendências globais, governamentais, da sociedade, dos setores privados e dos mercados. Por sua vez, o entrelaçamento de temas da saúde e do espaço urbano, até 2005 , consolidaria o campo de conhecimento da Saúde Urbana, que seria mais um apoio aos estudos de MA integrando o assunto com a pesquisa sistemática de indicadores e análise válidos, confiáveis e robustos guiados por métricas inovadoras para avaliar como intervenções na cidade podem impactar a saúde. (CAIAFFA; FRICHE; DANIELLE, 2015).

Ao propor aos indivíduos que priorizem, na medida do possível, os movimentos a pé pela cidade, aludindo a uma diversidade de vantagens tais como mais saúde, menos movimento de carros e poluição, mais economia e melhorias correlatas à ocupação, a segurança e a manutenção dos espaços públicos (MARQUET, 2015), a Mobilidade Ativa conseguiu agregar em torno de si atores que de alguma forma interessaram-se. No Brasil, sobretudo quando ganhou o status de Política para implantação do Sistema Nacional de Mobilidade Urbana através da Lei Federal 12.587 de 2012. Esta lei prevê que o Plano de Mobilidade Urbana deva estar compatibilizado com o plano diretor municipal existente ou em elaboração até 2018. Pode-se ser dito que nas esferas político-administrativas o debate sobre a Mobilidade Urbana aqueceu com o Sistema Nacional de Mobilidade, a pesquisa sobre a Mobilidade Ativa, por sua vez, parece ter recrudescido, no que tange ao estudo das correlações entre o espaço urbano e sua implementação. Poucos estudos têm se dedicado ao entrelace multidisciplinar, como se comentou inicialmente, sobretudo com o desenho urbano.

Grande parte das pesquisas atuais sobre a correlação entre cidades caminháveis, atividades físicas e saúde tendem a enfatizar variáveis demográficas e psicossociais (LESLIE et al., 2007, p. 113), tratando o 
espaço a partir de uns poucos atributos, fracos demais para estabelecer correlações ativas com outras variáveis. A exemplo disso, Oliveira-Brochado et al (2010, p. 7) enfatizam a necessidade de uma avaliação das influências psicossociais, culturais e das políticas de saúde sobre as atividades físicas do cidadão, visando forjar um "paradigma transdisciplinar". Mas para tal modelo, o espaço urbano é provedor passivo de atributos espaciais tais como a iluminação adequada, o clima adequado (arborização) e o conforto, todos esses mensurados a partir de atributos subjetivos, sem um planejamento espacial capaz de ativar positivamente o comportamento das pessoas.

Jeff Speck (2013) sistematizou recentemente sua teoria com base em três pontos de vista - que não se relacionam ao espaço propriamente dito, mas privilegiam as opiniões de economistas, epidemiologistas e ambientalistas. Muito embora busque uma teoria geral sobre a MA, estudando basicamente casos americanos, aquele autor propõe que os acessos urbanos tenham quatro condições básicas: utilidade, segurança, conforto e interesse. Desse modo, os movimentos da rotina diária individual devem ter seus pontos de início e fim próximos uns dos outros; os pedestres não podem ser ameaçados por automóveis; os edifícios e paisagens que limitam o espaço da rua devem constituir verdadeiros recintos urbanos e os passeios devem se alinhar a fachadas amigáveis, que se abram à atividades humanas. A partir dessas definições, aquele autor trata de enumerar dez passos para se alcançar a MA, descrevendo extensas recomendações pessoais baseadas em casos retirados de sua experiência profissional em cidades americanas.

Mais interessado no espaço urbano e ainda mais recente, o método de Christopher Leinberger e Rodriguez Michael "A Foot Traffic Ahead" (2016) ocupou-se em classificar trinta maiores áreas metropolitanas em diversas cidades dos EUA. Levando em consideração os limites territoriais das distintas regiões, calcularam seus indicadores econômicos e sociais. Espacialmente, tomaram as quantidades de áreas ocupadas por escritórios, comércios, moradias multifamiliares de cada setor e os compararam com o restante das demais regiões. Muitos ajustes metodológicos foram feitos no período de coleta de dados, que foi de 2007 a 2016 , e o principal deles foi o de considerar a renda familiar como um dos indicativos mais robustos para representar a produtividade e a equidade social, uma vez que esse dado Figura como um estado real de estabilidade das regiões estudadas. Isso indicava a hipótese de que as áreas caminháveis das cidades simultaneamente apresentavam uma maior renda, equidade social, e menor tendência a se desestabilizarem e se transformarem em outra coisa diante da especulação do mercado imobiliário. Aquele relatório apresentou uma bem ilustrada metodologia, e apontou ciclos de cidades de alta Mobilidade Ativa de acordo com diversos indicadores. Muito embora aparentasse ser um resultado óbvio para grandes aglomerações de alto nível socioeconômico, entreviu uma mudança do comportamento norte-americano relativamente ao espalhamento suburbano adotado há mais de sessenta anos, com urbanismo "arcadiano". O relatório demonstrou que novas métricas do adensamento e a inclusão de MA nas metrópoles eram capazes de qualificar a vida do cidadão, mais do que se pensara no passado. Similarmente, o relatório indicou uma diminuição do transporte pendular do trabalho nos centros urbanos às moradias nos subúrbios - esses últimos cada vez mais distantes - e a redução de transportes caros, causadores do aumento de poluição justamente nas áreas naturais, que estão cortadas por vias de alta velocidade. Se o "sonho americano" acreditou que o espalhamento suburbano e a manutenção de uma aparência de casas construídas em meio a paisagens naturais poderia mascarar os prováveis malefícios do espalhamento de baixa densidade, com esses novos estudos, ficou evidente que a melhor forma de preservar o ambiente natural seria ficarmos bem longe dele (MARQUET, 2015) e retornarmos às cidades para resolver seus problemas de habitação.

Outras abordagens conseguiram finalmente estudar o meio ambiente construído observando a variabilidade da ocorrência de certos elementos e condições espaciais e sociais que recursivamente se transformavam. Apresentam-se, dessa forma, como uma metodologia com a amplitude necessária para o estudo de correlações entre a saúde e o espaço urbano. Os "modelos ecológicos de comportamentos saudáveis" de Sallis Et al. (2008, p. 465) enfatizam agora que o ambiente e as ações políticas voltadas para a saúde devem ser estudados juntamente com as influências sociais, psicológicas e espaciais. Os modelos ecológicos conduziriam a uma pesquisa explícita sobre a recursividade dinâmica dos múltiplos níveis de influência que atuam no meio ambiente construído e nas formas sociais.

Um estudo bem mais robusto foi conduzido na Austrália e apresentado no artigo "Walkability of Local Communities: Using geographic information systems to objectively assess relevant environmental attributes (LESLIE Et al., 2007). Esta Pesquisa reconhece que os estudos sobre MA sempre partem da consulta da percepção psicossocial que as pessoas têm dos acessos na cidade, de modo extremamente empírico e subjetivo, através do já difundido NEWS e de outras iniciativas, como as ferramentas do site "Active Living Research" (2017). Ao contrário disso, este estudo sugere e justifica o uso de Sistemas Geográficos de Informação (SIG) para observar dados secundários sobre o espaço físico que permitam inferir sobre o maior ou menor uso dos acessos urbanos, anteriormente às entrevistas com os usuários. Dentre esses conjuntos 
de dados, estão a densidade populacional, o nível de conectividade das vias, a presença de redes formadas por conjuntos comerciais e de escritórios e a diversidade do uso da terra. A densidade parece óbvia, pois quanto mais gente, mais caminhadas haverá - esse é o princípio. A conectividade igualmente representa um acesso rápido entre as atividades rotineiras das pessoas, e quanto maior, mais representará as caminhadas úteis. Esclarecendo que o estudo se dedica a caminhadas que não sejam orientadas ao lazer, os autores acrescentam a importância da posição de escritórios e de concentrações comerciais, que são francos atratores de caminhantes, desde que estejam à uma distância razoável e competitível com automóveis, consequentemente desestimulante ao seu uso. Já a variedade de usos da terra corresponde e reforça a conectividade, adequando-se a um ritmo de vida cujo quotidiano se desenvolve em lugares variados. Aquele estudo deriva de uma pesquisa em comunidades australianas, e somente após a obtenção dos resultados das intercessões das camadas de informações secundárias em SIG é que se conduziu uma pesquisa quantitativa, entrevistando os caminhantes e confrontando as respostas e observações com o mapa de indicadores criados.

\section{ESTUDO DE CASO}

O último método mencionado na seção anterior é o que parece apresentar maior potencial para adaptar-se à criação de indicadores de MA para as cidades brasileiras. O desenho do estudo é ilustrado na Figura 1.

$O$ desenho do estudo levou em consideração, entretanto, um fato muito bem conhecido pelos pesquisadores que utilizam SIG no Brasil: a falta de qualidade dos dados (BARCELLOS; RAMALHO, 2002). Muito embora haja uma demanda crescente para a incorporação do SIG na pesquisa, a consolidação desse movimento depende ainda do acesso e qualidade dos dados, da existência de programadores e geômatas capazes de proverem algoritmos para a apreciação do cruzamento de variáveis entre camadas de informação, e da capacitação do pessoal e uma rede pública com interesses em democratizar informações e técnicas. Como se verá no estudo de caso aqui apresentado, tais fatores foram intervenientes e certamente indicam uma necessidade de melhoria em futuras pesquisas.

Figura 1: Desenho das etapas e processos para cálculo do MA no contexto da cidade de Belo Horizonte.

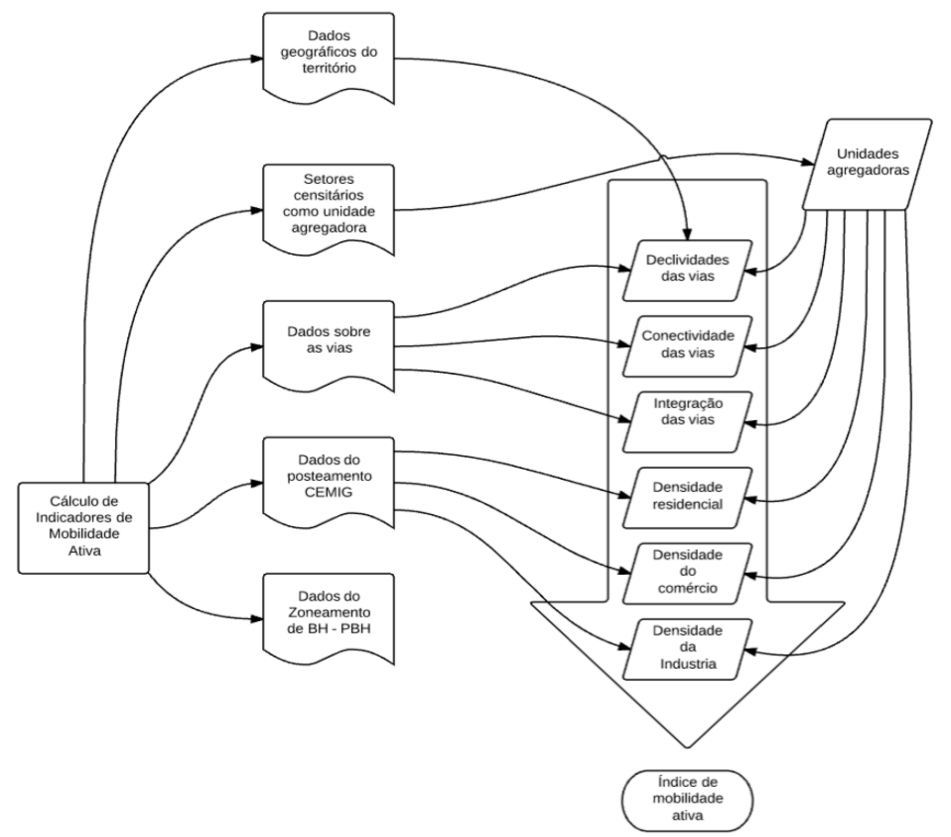

Fonte: O autor (2016).

Os dados foram obtidos do LabGeo, Laboratório de Geoprocessamento da Escola de Arquitetura da Universidade Federal de Minas Gerais. Apresentam-se aqui sem escala gráfica, mas integram-se pelos setores censitários. A maior parte deles provém do Censo 2010 (IBGE), excetuando-se os que foram obtidos por reinterpretação do mapa de kernel de posteamento da Companhia Energética de Minas Gerais (“Cemig", 2012). O mapa de kernel é uma alternativa para análise de padrões onde cada uma das observações é ponderada pela distância em relação a um valor central. Os dados do zoneamento de Belo Horizonte foram obtidos da Prefeitura municipal, mas, como se verá, não participam do cálculo. Os dados geográficos do território foram então representados em camadas e a unidade agregadora desses dados foram os setores censitários de 2010 (Figura 4). O software utilizado foi o Arcgis 10.2/2015. 


\section{Declividade das vias}

A declividade é interferente na MA uma vez que aclives e declives podem ser verdadeiros obstáculos para uma rota. O cálculo da declividade média de cada segmento das vias usou o modelo de cálculo explicado em Souza et al. (2016). O mapa da centerline (Figura 2a) teve cada um dos 49.885 segmentos de vias divididos em 3 pontos (Figura $2 b$ ) para garantir uma melhor estimação da declividade dos setores de via. A seguir, utilizando o mapa com as feições das curvas de nível do município, foi criado um mapa clinográfico (Figura 2c). A declividade de cada ponto dos segmentos foi capturada numa nova tabela e respectiva camada. Produziu-se, com isso, um mapa das vias de todo o município de Belo Horizonte (Figura 3), contendo o valor da declividade para os três pontos de cada segmento (início, meio e fim), dos quais se calculou a declividade média e sentido aclive/declive.

Figura 2: Divisões de segmentos viários em pontos sobrepostos no mapa clinográfico.

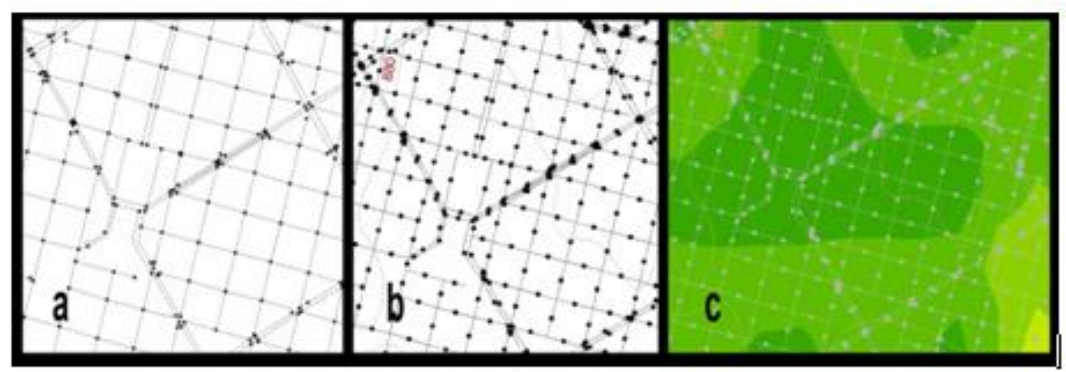

Fonte: O autor (2016)
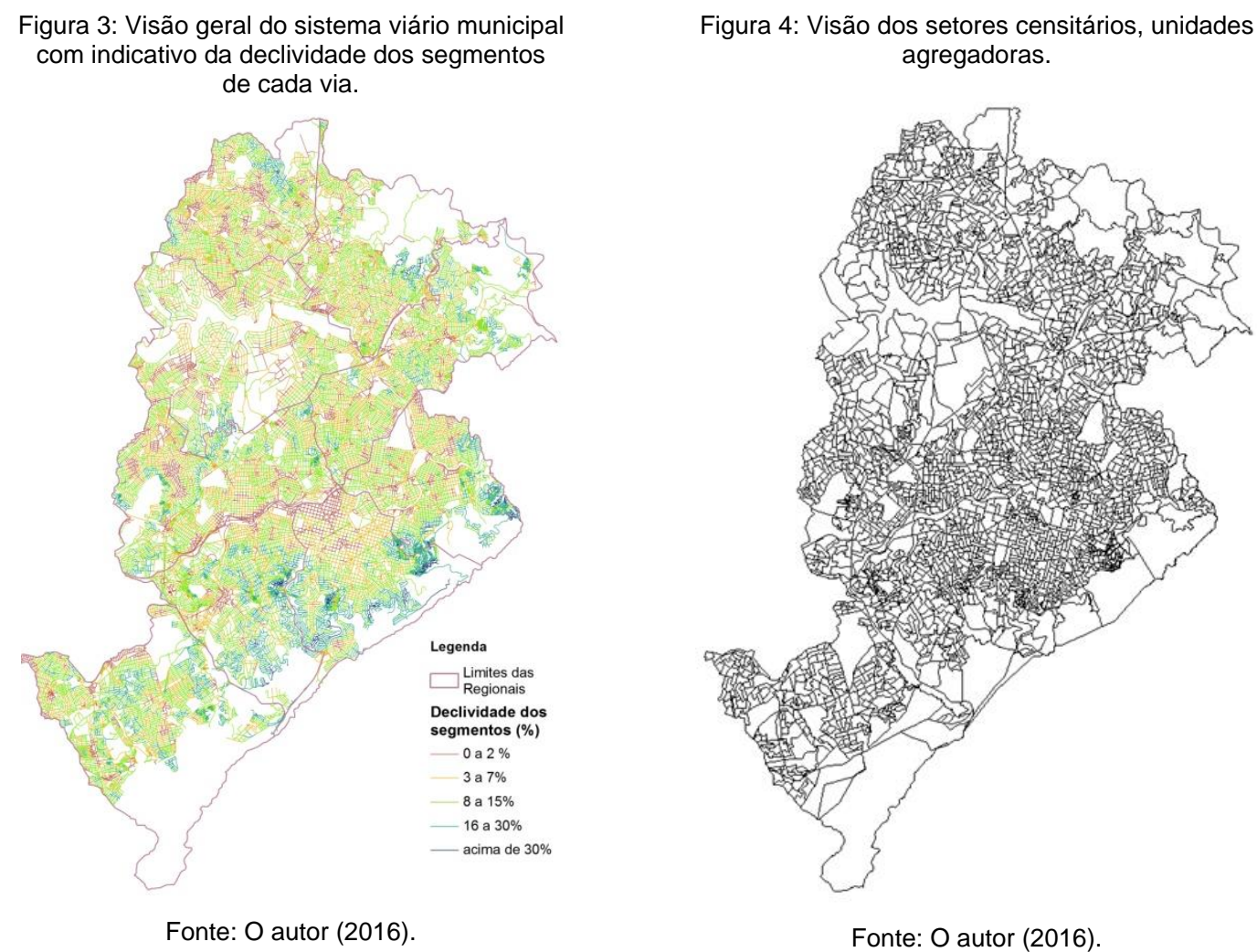

Fonte: O autor (2016).

Utilizou-se a seguir a média de declividade das vias contidas em cada setor censitário e os dados foram divididos em decis, que resultaram o mapa da Figura 5. Observe-se que no cômputo final, seus setores são subtraídos.

\section{Conectividade das vias: esquinas}

A conectividade das vias é indicadora da probabilidade de uso para a MA, de acordo com o número de segmentos de outras vias que a cortam. Aqui se descreve um dos processos utilizados. Em todos os segmentos viários as esquinas foram marcadas com um buffer suficientemente grande para se sobreporem a cada setor censitário. Caso se sobrepusesse sobre mais de um setor, era contado no total do respectivo setor, como mostra a Figura 6, onde os setores se representam por diferenças de cores enumeradas pelo 
número de esquinas contidas. O mapa resultante (Figura 7) mostra os decis relativos ao número de esquinas nas unidades agregadoras.

Figura 5: Decis de declividade viária do município.

Figura 6: Artifício para o cálculo de cruzamentos.
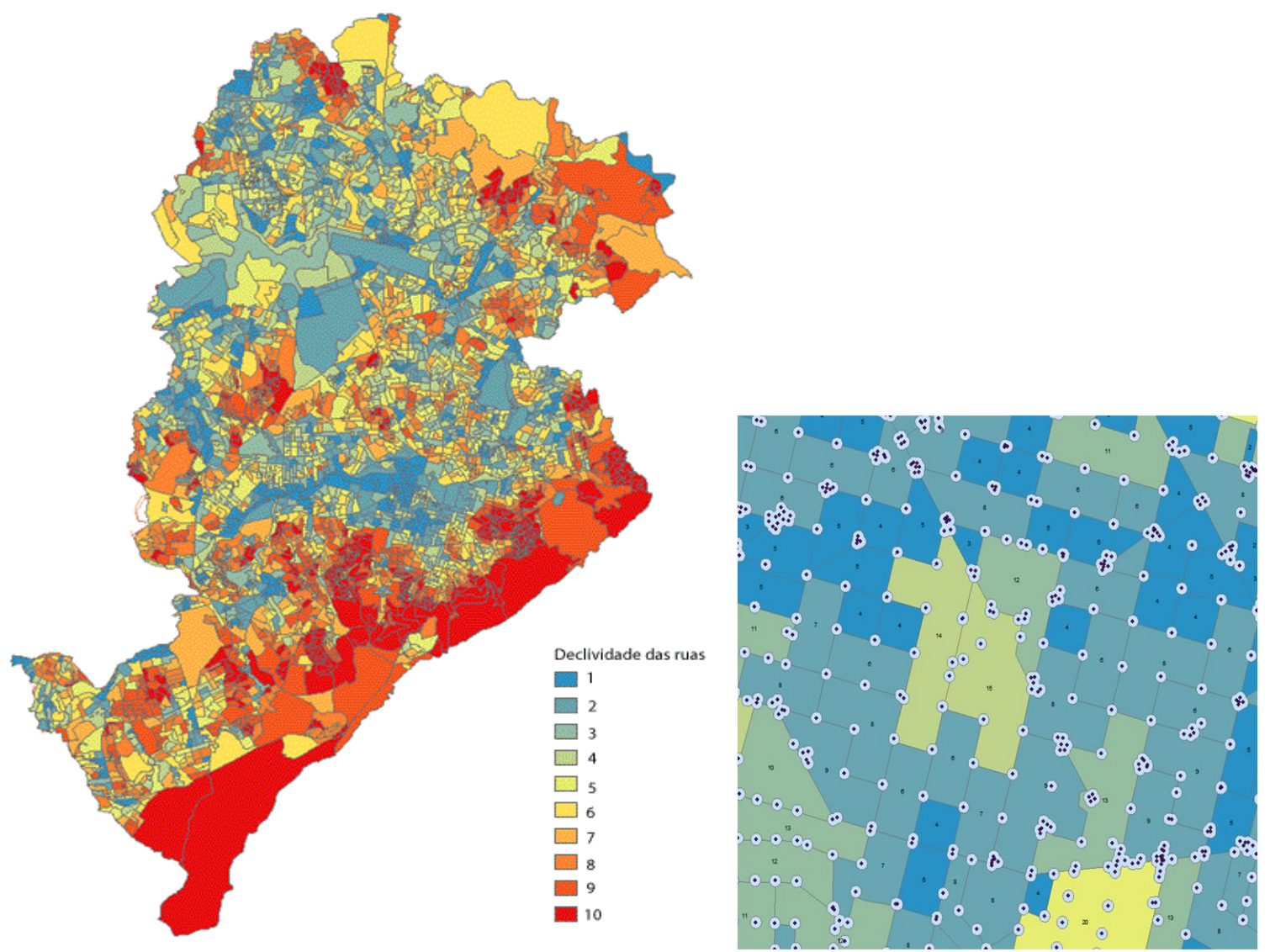

Fonte: O autor (2016).

\section{Conectividade das vias: integração e intermediação (choice)}

A teoria urbanística conFiguracional conhecida por Sintaxe Espacial (HILLIER, 1996; HILLIER; HANSON, 1984) propõe que uma via seja tratada como o eixo de um recinto urbano convexo, introduzindo, através do uso da teoria das redes, uma série de métricas que vêm sendo estudadas desde 1985, e têm apresentado sucesso na preditibilidade e solução de problemas urbanos em todo mundo. Para tanto, o sistema viário foi simplificado para sua alcançasse a axialidade das vias visando utilizar os cálculos propostos pela teoria.

O Software DepthMap@ (TURNER, 2013) foi utilizado para se calcularem conectividade, integração e intermediação, respectivamente. Conectividade ou integração local conta, desde uma esquina, quantas outras esquinas devem ser dobradas para que se alcance até mais duas. Integração global conta, com um raio infinito, não euclidiano e sobre as vias, quantas esquinas devem ser alcançadas desde cada esquina. Por sua vez, intermediação ou choice, ou ainda betweenness conta quantos menores percursos entre pares de segmentos de ruas passam por um dado segmento. Quanto maior sua intermediação, portanto, maior sua centralidade no sistema viário. Integração e intermediação podem ser somadas como medidas de centralidades dos espaços viários, sendo visualizadas como na Figura 7. A Figura 8 transfere para cada unidade agregadora a média do valor de integração e intermediação das vias contidas em cada setor censitário por hectare.

Como mencionado anteriormente, fatores atratores importam no estudo da MA. Entretanto, não houve possibilidade de se obter da Prefeitura Municipal um mapeamento de ocupação do solo segundo o uso proposto em lei. Leslie Et al (2007) propõe um método usando duas bases de dados, e interpretando sua junção com um fator aleatório, a título de fator de entropia. Utilizando o registro de impostos pagos sobre as mercadorias e serviços, soma-os aos impostos do zoneamento urbano. Tal método mostrou-se inviável pelo tempo que iria requerer. 
Figura 7: Mapa de decis de esquinas por hectare nas unidades censitárias.

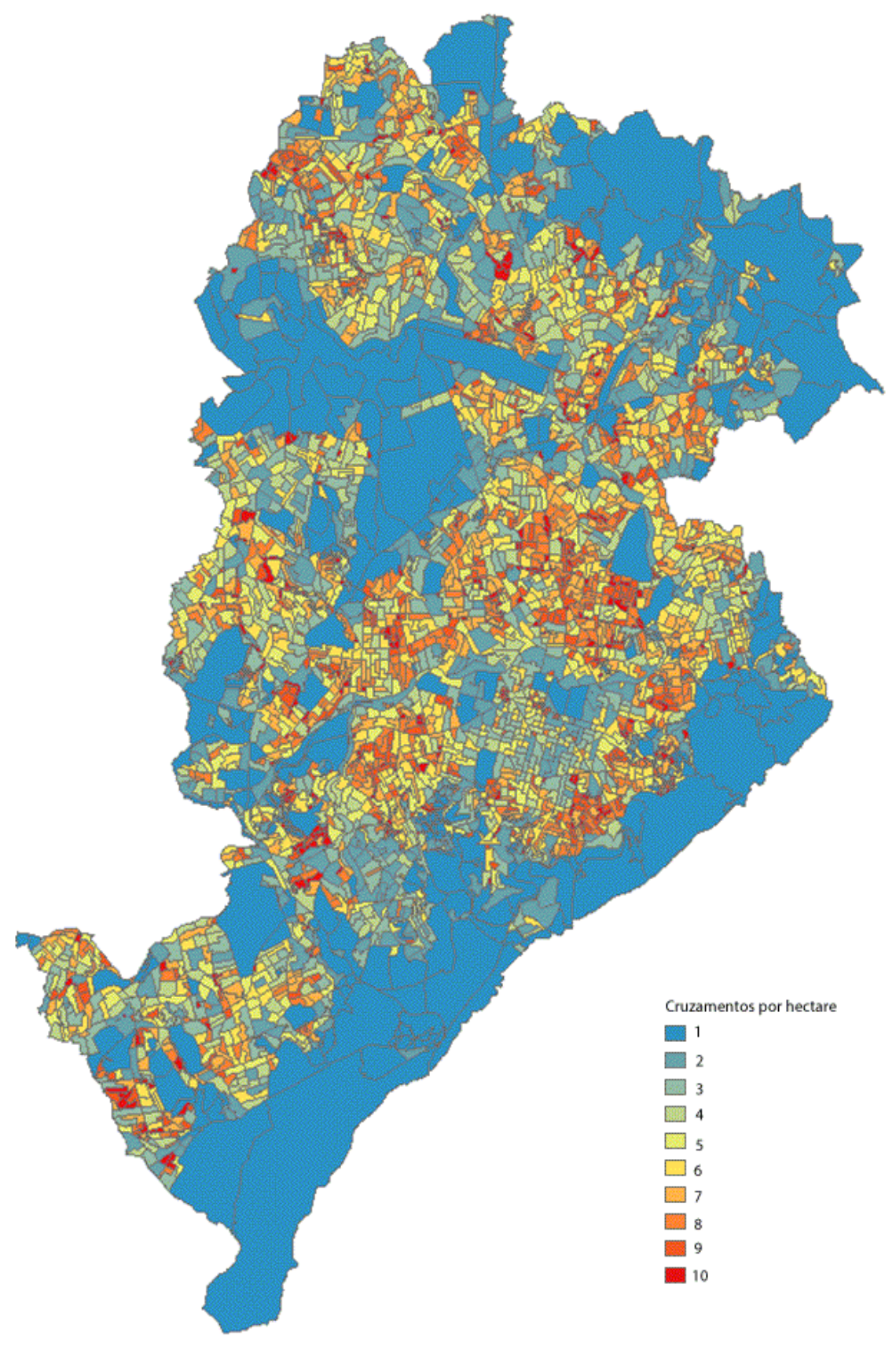

Fonte: O autor (2016).

Dessa maneira, adotou-se a estratégia de utilizar os dados para o Cálculo da densidade da indústria, comércio e de residências do município a partir do levantamento do posteamento da CEMIG 2012, que os localiza, designando-os por esses três tipos de uso. A base de dados foi cedida pelo LABGEO - EAUFMG, na forma de um mapa de densidades de kernel, sobre o município. Os mapas correspondentes para o cálculo dos decis foram executados de acordo com a densidade média retirada dos mapas de kernel e mostram-se nas Figuras 10, 11 e 12. 
Figura 8: Integração e Intermediação global das vias (INCH).

(Quanto mais quente a cor, mais integrada e próxima topologicamente de todas as outras vias).

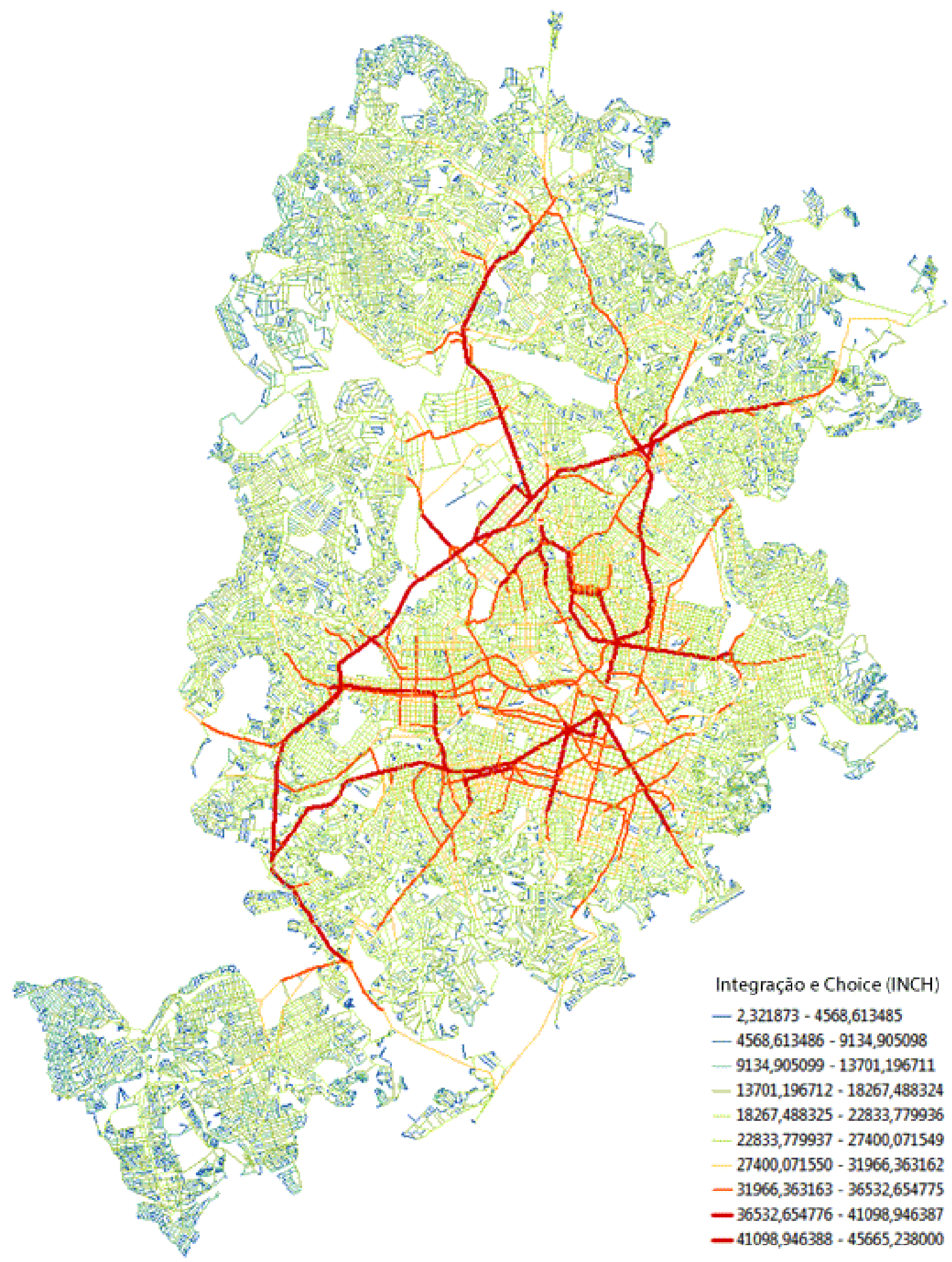

Fonte: O autor (2016) 
Souza, R. C. F.

Figura 9: Valor médio de INCH transposto por HA no setor censitário.

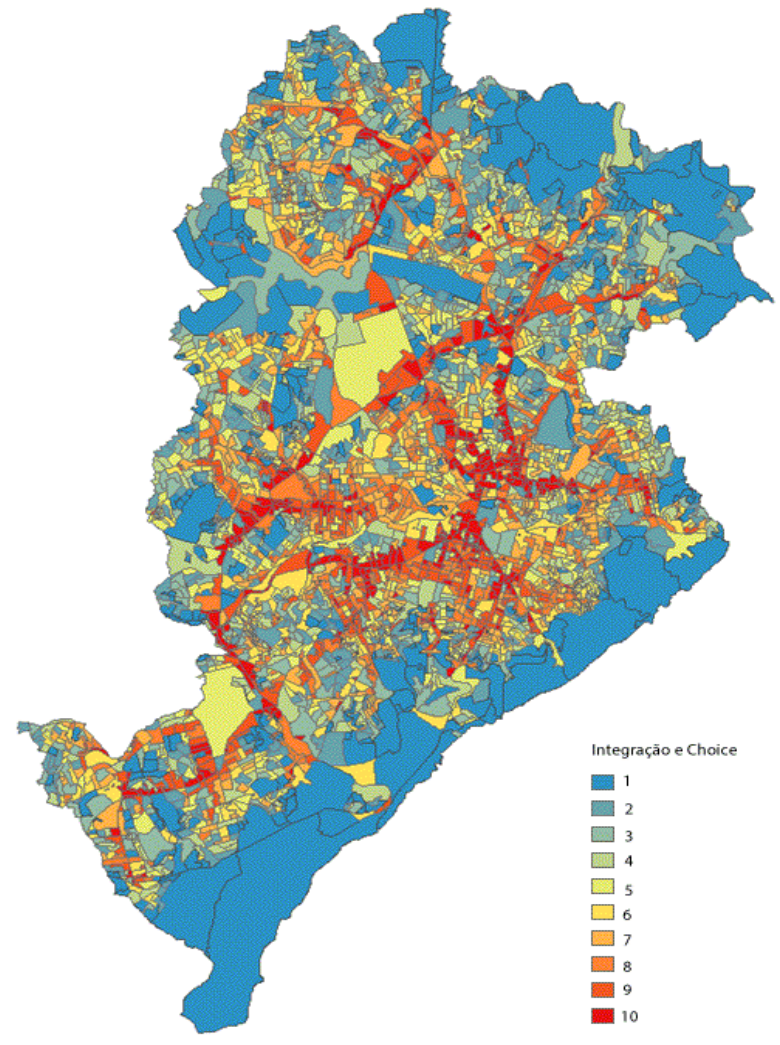

Figura 10: Densidade do comércio por setor.

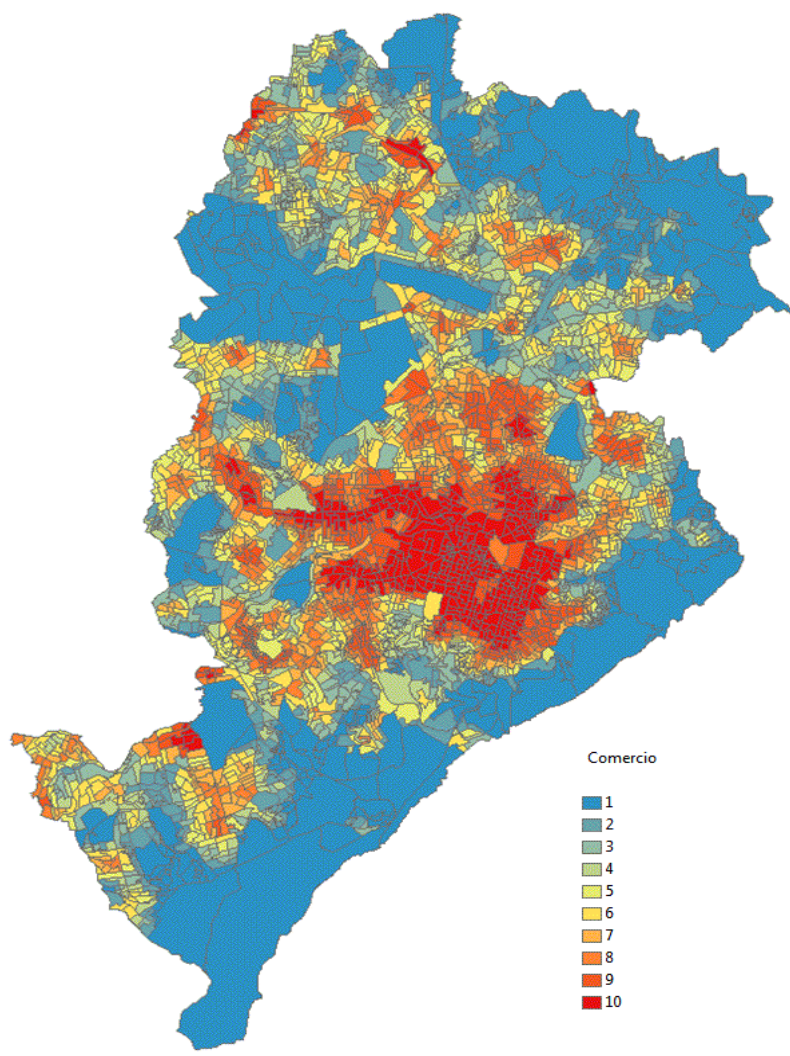

Fonte: O autor (2016).

Figura 11: Densidade da indústria por setor.

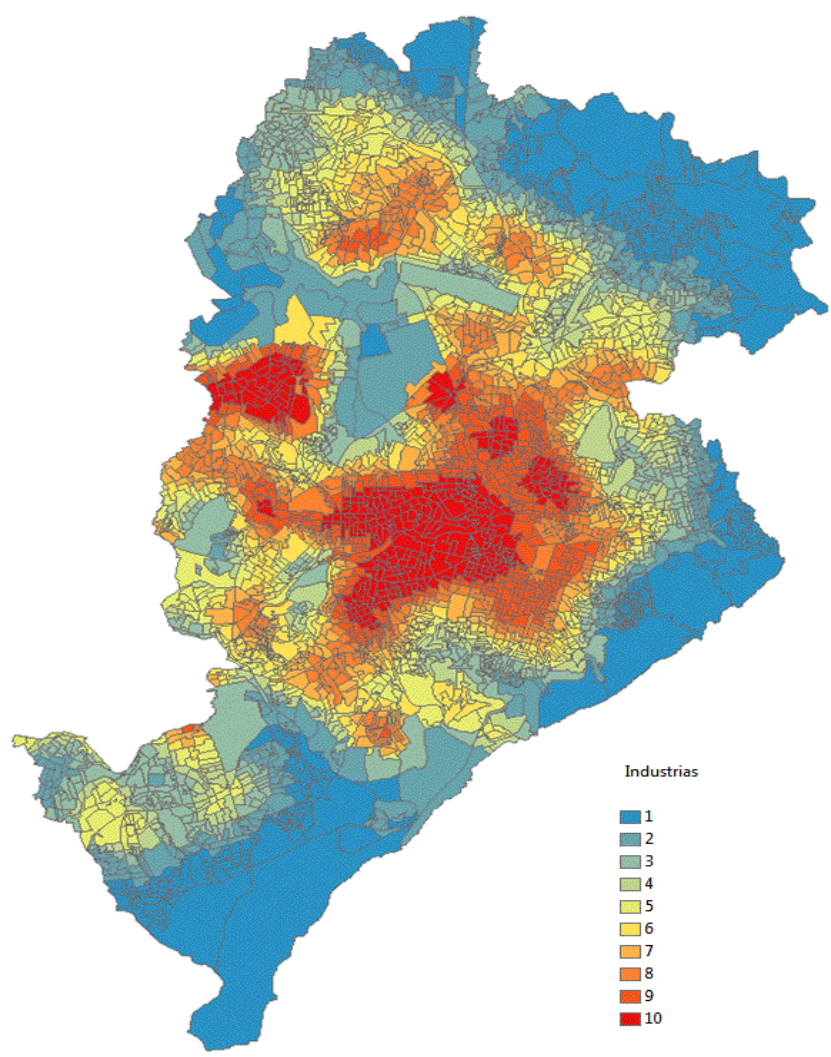

Figura 12: Densidade de residências por setor.

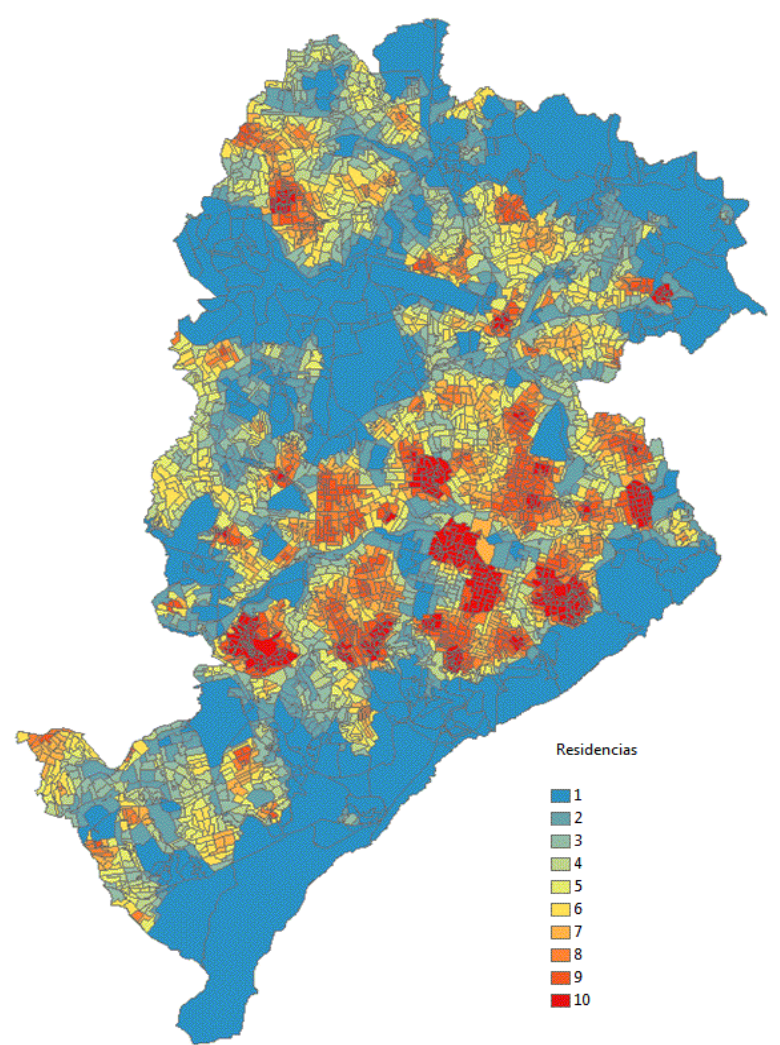

Fonte: O autor (2016). 


\section{0 cálculo Final do indicador de Mobilidade Ativa}

Uma vez que cada unidade agregadora de cada camada teve o cálculo de valores em decis, a última etapa foi juntar, no ArcMap@ as camadas e respectivos setores, calculando sua média. Lembrando que, na Figura 13, a primeira camada traduz os segmentos de rua e sua declividade, a segunda, a conectividade por cruzamentos, a terceira a conectividade conFiguracional (integração e intermediação das vias), a quarta a densidade habitacional por setores, a quinta é a densidade comercial e a sexta a densidade industrial. A sétima camada é o mapa resultante dos indicadores finais de Mobilidade Ativa, na Figura 13.

Figura 13: Esquema de cálculo final de indicador de MA - cálculo da média dos setores.

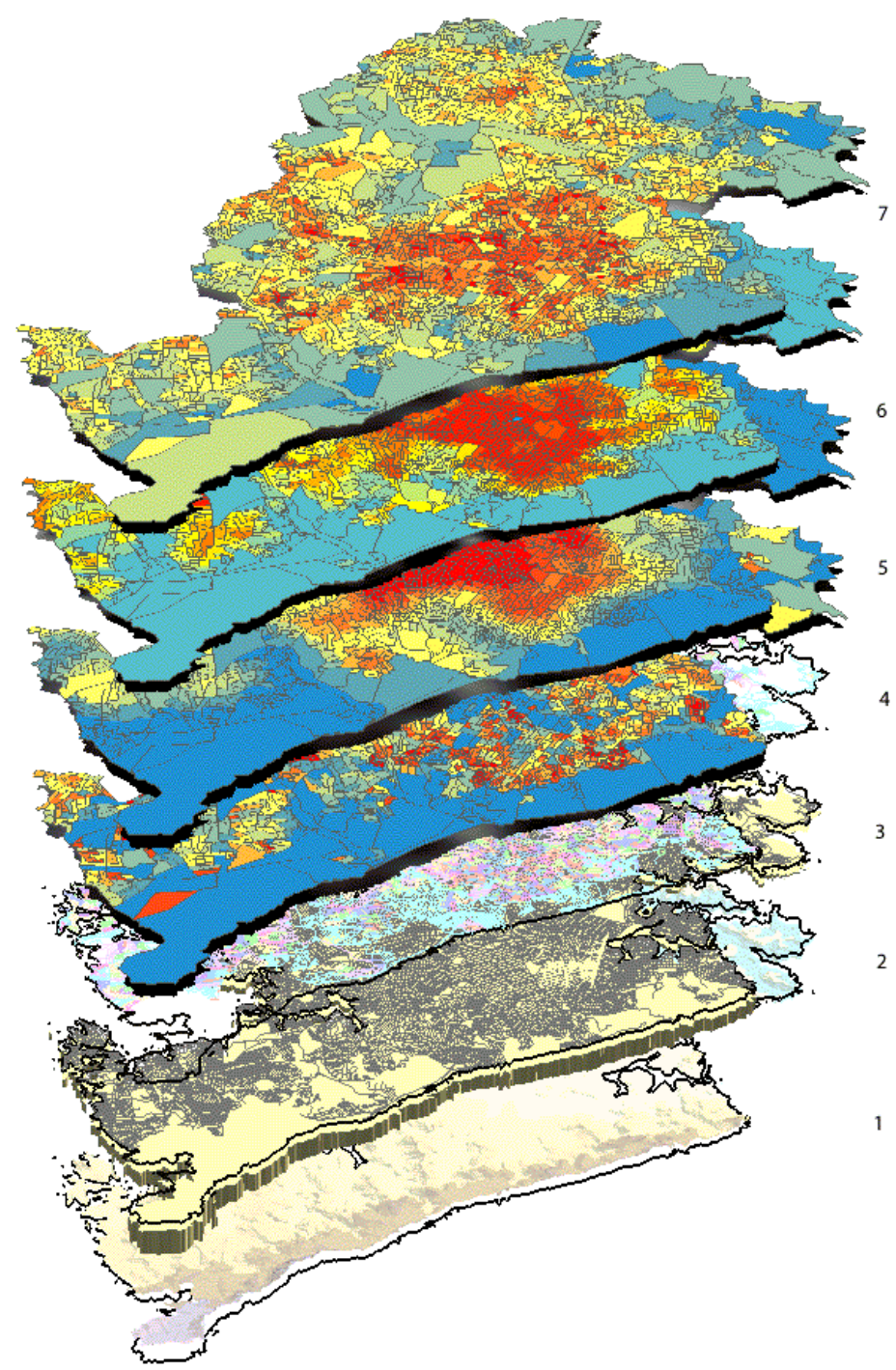

Fonte: O autor (2016)

\section{ANÁLISE}

Uma vez elaborado o mapa, seguiu-se uma seção analítica prévia do resultado. Os índices de Mobilidade Ativa foram classificados por cores mais frias, indicando baixos índices, até mais quentes, altos índices. Várias outras camadas com informações municipais foram sobrepostas, e a estas, outras ainda do Censo 2010 (IBGE, 2012).

Como se mencionou anteriormente, esse experimento metodológico teve por finalidade reduzir o questionário de Brian Saeles e James Sallis (2002), o NEWS e suas adaptações posteriores, permitindo obter maior precisão nas questões que fossem reveladoras da percepção dos moradores de cada região. A pesquisa ainda está em curso, e as análises básicas permitem inferir que não existe uma correlação imediatamente perceptível entre a vivência da cidade e o índice. Dito de outra maneira, índices altos de MA 
não indicam boa qualidade da experiência do pedestre, e podem cobrir vias e passeios com acessibilidade comprometida pela preferência dada aos automóveis. Igualmente, baixos índices podem ocorrer em regiões onde intuitivamente localizam-se bons passeios, praças, academias da cidade, pistas de cooper e ciclovias

Figura 14: Índice de Mobilidade Ativa para o município de Belo Horizonte.

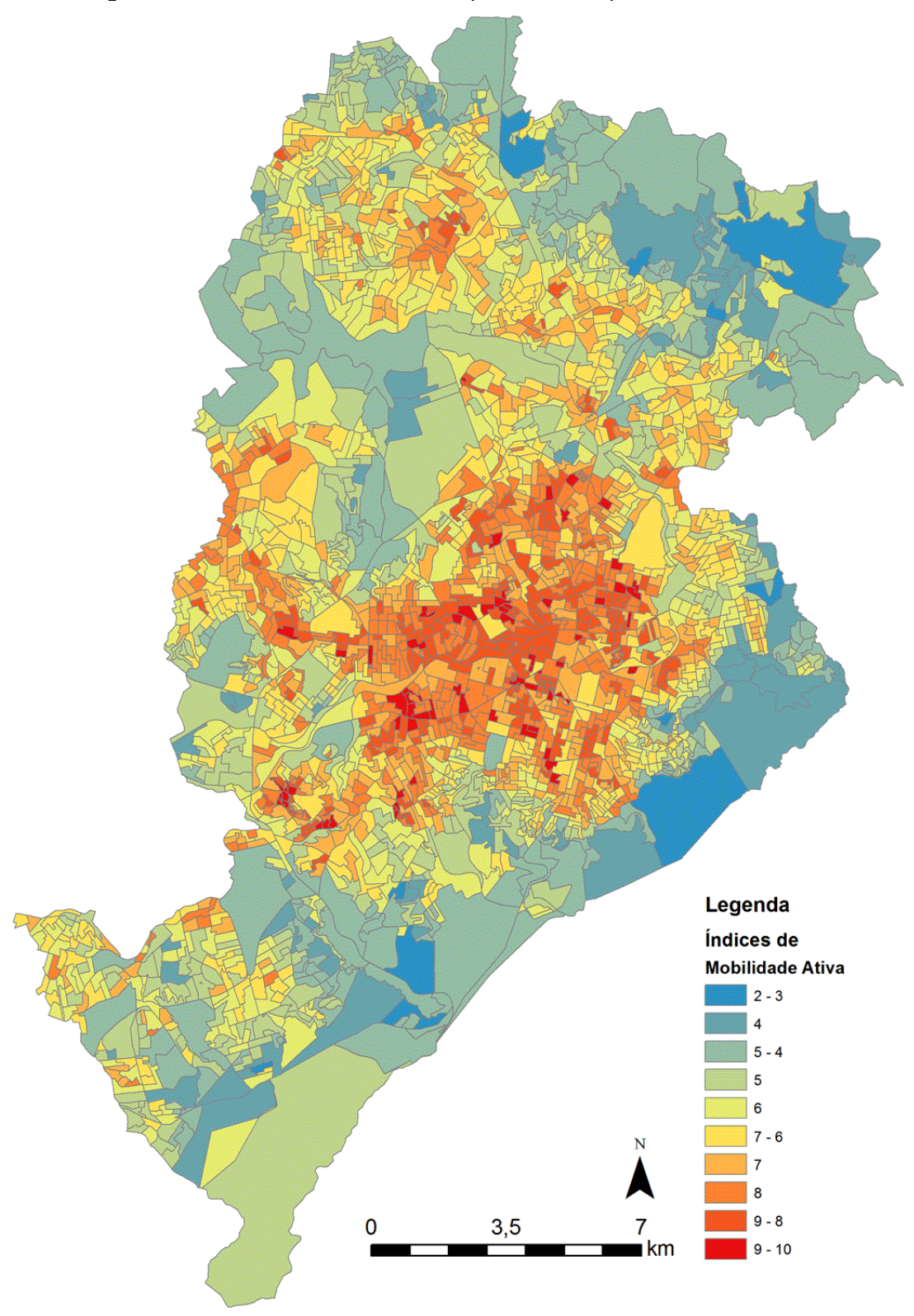

Fonte: O autor (2016).

Três aspectos foram notórios nessa análise prévia. Ao contrário dos resultados do método de Christopher Leinberger e Rodriguez Michael (2016), que consideram a renda familiar como indicativo para representar a produtividade e a equidade social na hipótese que áreas mais ricas que têm maiores índices de MA, os resultados para Belo Horizonte demonstram que as áreas de maior renda possuem baixos índices, mas grandes áreas de baixa renda, extremamente vulneráveis à especulação do mercado imobiliário, também tem esses índices baixos.

Crianças menores de 10 anos e idosos maiores que 65 anos se espalham com certa homogeneidade nas áreas de mais baixos índices de mobilidade, deixando entrever a necessidade de repensar o acesso dessa população às áreas públicas da cidade.

Finalmente, outro aspecto chamou a atenção: áreas que passaram por reformas urbanas e implantação de conjuntos habitacionais desenvolveram características diferentes dependendo do índice de integração. As três ocupações urbanas subnormais mostradas em destaque na Figura 15 exemplificam esse fenômeno: as comunidades Pedreira Prado Lopes, do Complexo da Serra e da Vila CEMIG. 
Figura 15: Estudos iniciais de comunidades de assentamento urbano subnormal.

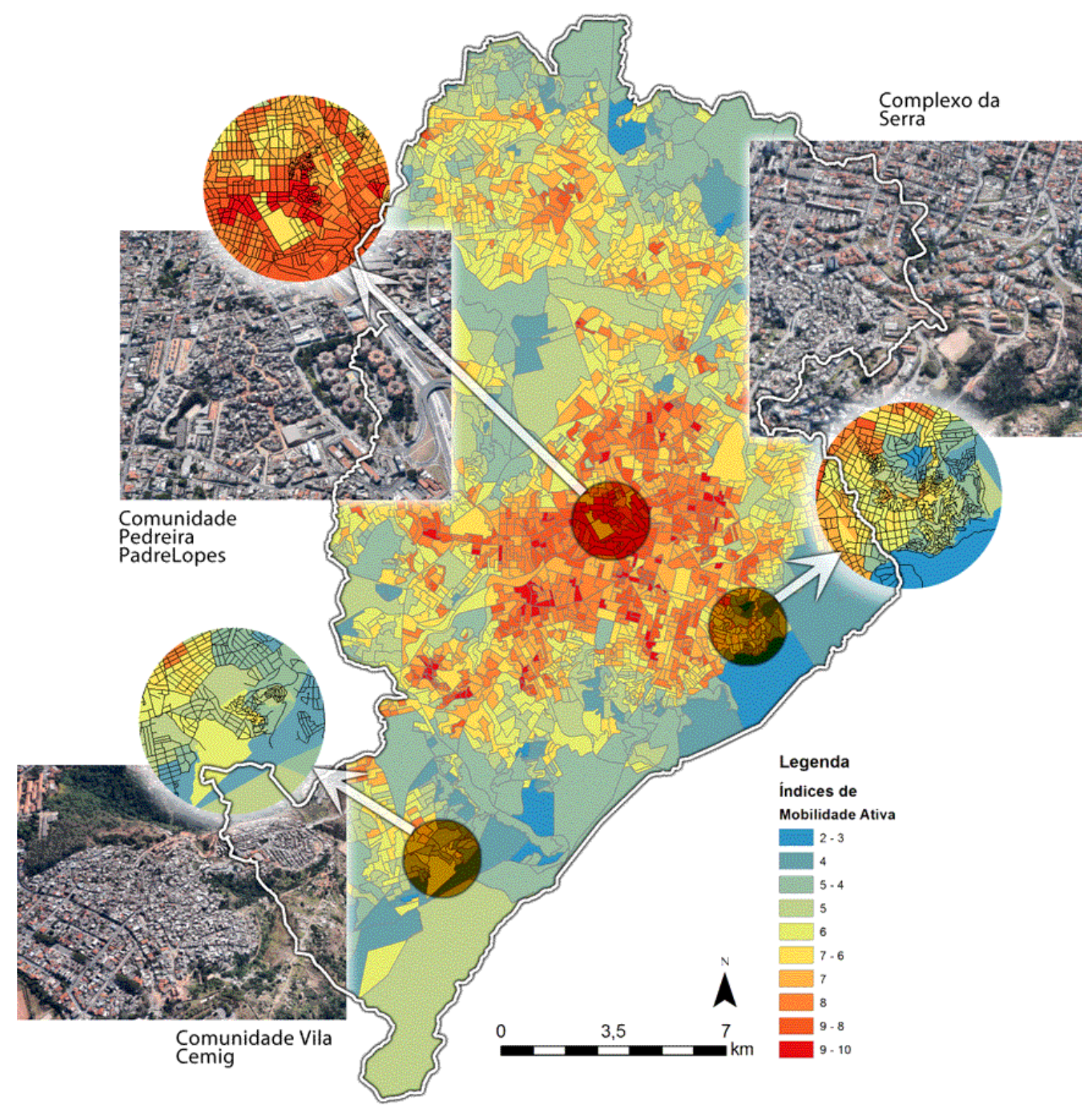

Fonte: O autor (2016).

- A Comunidade Pedreira Prado Lopes concentrou um dos maiores índices nacionais de criminalidade entre os anos de 1998 e 2002 (LIMA, 2010) e recentemente tornou-se integrada por meio de uma alta Mobilidade Ativa, causada pela implantação de um conjunto habitacional do programa Vila Viva.

- As comunidades do Complexo da Serra, onde também recentemente o programa Vila Viva implantou conjuntos habitacionais e refez espaços públicos, quadras, praças e passeios; a integração das vias proporcionada pelos projetos causou uma difusão de índices de MA altos e baixos; dados atuais mostraram pequena redução da criminalidade.

- A Comunidade Vila CEMIG, talvez seja a mais desassistida e com baixa MA; seus índices se preservam, e acolhe grande quantidade de crianças e idosos.

\section{CONCLUSÃO}

O método testado apresentou um grande número de possibilidades para adaptar qualquer método de observação social sistemática, introduzindo complexidades locais que poderiam ser relatadas apenas como subjetividades incomensuráveis (EWING; HANDY, 2009). Não se apresentou como um mapa com sua própria e lógica reserva sintagmática para entendimento, mas acentuou discrepâncias não explicadas pela simples sobreposição de camadas censitárias. No momento, a pesquisa está em curso e uma série de 
testes vem sendo feitos relativamente às relações da MA e melhoria da saúde urbana, do ambiente, das economias locais, da poluição em geral, e do fortalecimento dos programas para reapropriação dos espaços públicos das cidades.

O método mostrou a possibilidade de discutir a criminalidade (NUBANI; WINEMAN, [s.d.]) e a questão das transformações dinâmicas dos assentamentos urbanos subnormais como polos de segregação e de integração socioespaciais (1984), no caso, visíveis através da MA. A forma ou a organização espacial de uma sociedade pode ser vista como uma função das diferentes formas de solidariedade social, e nos espaços socialmente segregados, os encontros e interações resultantes da proximidade física e do compartilhamento de categoria ou classe se reforçam mutuamente, à custa das relações com membros de outros grupos. Já quando há a integração socioespacial, as relações entre indivíduos diferentes ocorrem localmente em função da proximidade e das possibilidades de encontro, ao passo que globalmente ocorre a interação propiciada pelas afinidades de classe ou categoria. Esse sistema baseado na segregação socioespacial precisa, para se reproduzir, de restrições a encontros e de regras e limites espaciais muito fortes, tendendo à exclusividade e, no longo prazo, ao fortalecimento do grupo local em detrimento do sistema global, diminuindo sua MA. Já no sistema sócio espacialmente integrado, a manutenção da diversidade depende da não exclusividade, as regras e limites precisam ser mais fracos, com uma abertura nas relações entre habitantes e entre habitantes e estranhos, tendendo, assim, a reforçar tanto o sistema global quanto o grupo local.

Atualmente, pesquisa-se sobre a possibilidade de criar índices de MA em cidades com pouca ou nenhuma informação sistematizada em SIG. Lança-se mão do sistema de vias destas cidades, oferecidas pelo OSM (OPENSTREETMAP, 2016), utilizando-se a Análise Sintática (JIANG; CLARAMUNT, 2002) e verificando-se como as adaptações, quando coerentes com o conhecimento metodológico, permitem apoiar decisões da gestão e da fruição dos espaços urbanos. Pesquisa-se também, para a cidade de Belo horizonte, o conceito de vitalidade urbana e seus índices, posto que altos índices de MA marcaram-se em espaços perigosos e desqualificados para pedestres.

\section{REFERÊNCIAS}

BARCELLOS, C. DE C.; RAMALHO, W. M. Situação atual do geoprocessamento e da análise de dados espaciais em saúde no Brasil. Informática pública, v. 4, n. 2, p. 221-230, 2002.

CAIAFFA, W. T.; FRICHE, A. A. DE L.; DANIELLE, C. Urban health: landmarks, dilemmas, prospects, and challenges. Cadernos de Saúde Pública, v. 31, pp. 5-6, 2015.

COMPANHIA ENERGÉTICA DE MINAS GERAIS (CEMIG).site Disponível em: <http://www.cemig.com.br/pt$\mathrm{br} /$ Paginas/default.aspx>. Acesso em: 14 out. 2016.

INSTITUTO BRASILEIRO DE GEOGRAFIA E ESTATíSTICA (IBGE). CENSO 2010. Disponível em: [www. ibge. gov. br/home/estatistica/populacao/censo2010/]. Acessado em 14 de out 2012.

EWING, R.; HANDY, S. Measuring the Unmeasurable: Urban Design Qualities Related to Walkability. Journal of Urban Design, v. 14, n. 1 (1), pp. 65-84, fev. 2009.

HILLIER, B. Space is the machine: a conFigurational theory of architecture. Cambridge: Cambridge University Press, 1996.

HILLIER, B.; HANSON, J. The social logic of space. Cambridge: Cambridge University Press, 1984.

JIANG, B.; CLARAMUNT, C. Integration of Space Syntax into GIS: New Perspectives for Urban Morphology. Transactions in GIS, v. 6, n. 3 (1), pp. 295-309, jun. 2002.

LEINBERGER, C. B.; MICHAEL, R. Foot Traffic Ahead - Smart Growth America. The Center for Real Estate and Urban Analysis, v. 01, n. 01, 2016, p. 40.

LESLIE, E. Et al. Walkability of local communities: using geographic information systems to objectively assess relevant environmental attributes. Health \& place, v. 13, n. 1, pp. 111-122, mar. 2007.

LIMA, A. V.; RECH, C. R.; REIS, R. S. Equivalência semântica, de itens e conceitual da versão brasileira do Neighborhood Environment Walkability Scale for Youth (NEWS-Y) Semantic, item, and conceptual equivalence of the Brazilian version of the Neighborhood Environment. Cadernos de Saúde Pública, v. 29, n. 12, pp. 2547-2553, 2013.

LIMA, V. C. DE S. Espaço e Criminalidade em Favelas de Belo Horizonte: um estudo sob a ótica da segregação e do controle socioespacial. Belo Horizonte: Universidade Federal de Minas Gerais, 2010.

LUFKIN, B. Are walkers smarter than drivers? Disponível em: <http://www.bbc.com/autos/story/20160712-do-smartpeople-walk-more>. Acesso em: 15 jul. 2016. 
MALAVASI, L. M.; F.S.DUARTE, M. F. S.; BOTH, J. Neighborhood Walkability Scale (news - Brazilian Version): Construct Validity. Medicine \& Science in Sports \& Exercise, v. 39, n. Supplement, pp. 180/190, maio 2007.

MARQUET, O. Speck, Jeff (2012). Walkable City: How Downtown Can Save America, One Step at a Time. Documents d'Anàlisi Geogràfica, v. 61, n. 2, p. 436, 19/maio/2015.

NUBANI, L.; WINEMAN, J. The Role of Space Syntax in Identifying the Relationship Between Space and Crime. University of Michigan [s.d.].

OLIVEIRA-BROCHADO, A.; OLIVEIRA-BROCHADO, P.; BRITO, P. Q. Effects of personal, social and environmental factors on physical activity behavior among adults. Epidemiologia Básica da Escola Nacional de Saúde Pública de Lisboa, v. 28, n. 01, p. 7-17, 2010.

OPENSTREETMAP. Disponível em: <https://www.openstreetmap.org/search?query= Belo\%20Horizonte\#map=12/19.9181/-43.9603 >. Acesso em: 12 out. 2016.

RESEARCH, A. L. Active Living Research. Disponível em: <http://activelivingresearch.org/ toolsandresources/all>. Acesso em: 16 jun. 2017.

SAELENS, B. E.; SALLIS, J. F. Neighborhood Environment Walkability Survey - NEWS-A. [s.I.] Measurement Instrument Database for the Social Science, 2002. Disponível em: <www.midss.ie>. Acesso em 16 de junho de 2016

SALLIS, J. F. Neighborhood Environmental Walkability Scale. Disponível em: $<$ http://sallis.ucsd.edu/measure_news.html>. Acesso em: 13 jun. 2017.

SALLIS, J. F.; OWEN, N.; FISHER, E. B. Ecological Models for Health Behaviour. In: K. GLANZ; B. K. RIMER; K. VISWANATH (Ed.). Health behavior and health education: theory, research, and practice. San Francisco, CA: JosseyBass, pp. 465-485, 2008.

Shay, Elizabeth; Spoon, S; Khattak, A; Center, S T. Walkable environments and walking activity. Final Report for Seed Grant Submitted to Southeastern Transportation Center, University of Tennessee, 2003.

SOUZA, R. C. F.; OLIVEIRA, V. B.; PEREIRA, D. B.; COSTA, H. S. M.; CAIAFFA, W.T. Viver próximo à saúde em Belo Horizonte. Cadernos Metrópole, v. 18, n. 36, pp. 326-344, $2016 . \quad$ Disponível em: http://www.redalyc.org/articulo.oa?id=402846273002. Acesso em 12 de janeiro de 2016.

SPECK, J. Walkable city. In: Ted Talk Ideas Worth Spreading, set/2013. Disponível em: <https://www.ted.com/talks/jeff_speck_the_walkable_city/transcript?language=en\#t-980729>. Acesso em 12 de janeiro de 2016.

TURNER, A. Depthmap. UKUniversity College of London, 2013. Disponível em: $<$ http://www.spacesyntax.net/software/ucl-depthmap/>.

WHO. WHO 2002: move for health. World Health Day, 7/dez/2010.

NOTA DO EDITOR (*) O conteúdo do artigo e as imagens nele publicadas são de responsabilidade do(s) autor(es). 